\title{
COMPOSIÇÃO FLORÍSTICA DE UMA FLORESTA SEMIDECÍDUA MONTANA, NA SERRA DE SÃO JOSÉ, TIRADENTES, MINAS GERAIS
}

\author{
Ary Teixeira de Oliveira-Filho ${ }^{1}$ \\ José Nivaldo de Menezes Machądo ${ }^{2}$
}

Recebido em 16-12-92. Aceito em 09-09-93.

RESUMO - (Composição florística de uma floresta semidecídua montana na Serra de São José, Tiradentes, Minas Gerais). Um levantamento florístico das espécies arbustivas e arbóreas (diâmetro na base do caule $>=5 \mathrm{~cm}$ ) foi realizado na floresta semidecídua montana que ocorre nos contrafortes da serra de São José, Tiradentes, MG. 0 levantamento incluiu as espécies encontradas no interior de 95 parcelas de $10 \times 10 \mathrm{~m}$ bem como aquelas coletadas fora da área amostral. É apresentada uma relação de 277 espécies pertencentes a 67 famílias botânicas. A presente listagem é comparada quantitativamente com outros levantamentos florísticos realizados nos estados de Minas Gerais e São Paulo.

Palavras-chave: Composição florística, floresta semidecídua montana, Minas Gerais, Brasil.

ABSTRACT - (Floristic composition in a semideciduous montane forest in the Serra de São José, municipality of Tiradentes, Minas Gerais State). A floristic survey of the shrub and tree species (diameter at the base of the stem $>=5 \mathrm{~cm}$ ) occurring in a semideciduous montane forest was carried out in the foothills of the Serra de São José, municipality of Tiradentes, state of Minas Gerais, Brazil. The survey included all species found in 95 quadrats of $10 \times 10 \mathrm{~m}$ as well as those collected outside the sample area. A list of 277 species belonging to 67 plant families is presented. The present checklist is compared quantitavely to other floristic surveys carried out in the states of Minas Gerais and São Paulo.

Key-words: Floristic composition, semideciduous montane forest, Minas Gerais, Brazil.

\section{Introdução}

Na cobertura vegetal primitiva do estado de Minas Gerais as fisionomias florestais predominavam em uma vasta região correspondente ao centro-sul e ao leste do estado

1 - Bolsista do CNPq, processo $\mathrm{n}^{\circ}$ 301644/88-8. Departamento de Ciências Florestais. Escola Superior de Agricultura de Lavras (ESAL). 37200 Lavras, MG, Brasil.

2 - Chefe da Estação Florestal Experimental (EFLEX) do IBAMA, Ritápolis, MG. 
(IBGE, 1984). Da mesma forma como ocorreu para vários outros estados brasileiros onde o processo de ocupação e exploração remonta o período colonial, nesta região de Minas Gerais, a cobertura florestal primitiva foi reduzida a remanescentes esparsos, sendo que a maioria deles encontra-se ou bastante perturbada pela retirada seletiva de madeira ou situada em áreas onde a topografia dificulta 0 acesso.

Apesar da crescente consciência sobre a importância destes recursos florestais e sobre a urgência de conservá-los, pouco se sabe sobre a composição florística dos remanescentes florestais existentes no centro-sul e leste de Minas Gerais. Estudos pioneiros têm recentemente resultado em uma série de levantamentos florísticos de algumas cadeias de montanhas da região, cuja vegetação inclui relictos florestais: maciço do Caraça (Ferreira \& Magalhães, 1977), serras do Grão Mogol e Ibitipoca (Ferreira et al., 1977/78), serra da Piedade (Brandão \& Gavilanes, 1990), serra do Carrapato (Gavilanes et al., 1992a), serra do Curral (Brandão, 1992). As recentes iniciativas visando a recuperação de matas ciliares resultaram igualmente em uma série de levantamentos de matas ciliares na região do alto rio Grande (Carvalho et al., 1992; Gavilanes et al., 1992b; Vilela et al. 1993). Contudo, o volume de informações florísticas é ainda pequeno, o que fica evidenciado quando se compara ao que já foi acumulado sobre os remanescentes florestais do estado de São Paulo (vide revisões em Meira Neto et al., 1989 e em Torres et al., 1993).

Além das alardeadas justificativas para a conservação das florestas remanescentes baseadas na preservação da diversidade genética e na importância para outros recursos naturais, como solo, água e fauna, o valor paisagístico é particularmente crucial em regiões onde a atividade turística desempenha um forte papel econômico. Este é o caso da cidade de Tiradentes, onde a serra de São José forma um espetacular cenário de fundo para o casario colonial, com suas escarpas de rocha nua assentadas sobre uma encosta inteiramente revestida por uma impressionante floresta. Apesar de sua reconhecida importância para a comunidade, esta floresta vem sendo cada dia mais ameaçada por atividades como a extração de lenha e as queimadas. As preocupações com a conservação dos recursos naturais da serra de São José têm sido canalizadas pela Sociedade Amigos de Tiradentes (SAT) que, em 1991, obteve do governo estadual o status de Área de Preservação Ambiental (A.P.A.) para boa parte do conjunto da serra. Como a condição de A.P.A. prevê o uso regulado da terra pelos seus proprietários, a SAT tem procurado descobrir e desenvolver métodos não destrutivos de uso dos recursos florestais que sejam aplicáveis à região.

Desta forma, tornou-se evidente que o conhecimento da flora era fundamental para o desenvolvimento de quaisquer estratégias de ação, além de evidenciar o valor em biodiversidade da vegetação nativa. Atendendo solicitações da SAT à Estação Florestal Experimental (EFLEX) do IBAMA, em Ritápolis (MG), e à Escola Superior de Agricultura de Lavras (ESAL), realizamos um levantamento florístico do componente arbustivoarbóreo da floresta que reveste os contrafortes da serra de São José, cujos resultados são apresentados na presente contribuição.

\section{Material e métodos}

A serra de São José está situada no centro-sul do estado de Minas Gerais, entre as coordenadas geográficas de $21^{\circ} 00^{\prime}$ a $21^{\circ} 02^{\prime}$ de latitude sul e $44^{\circ} 00^{\prime}$ a $44^{\circ} 15^{\prime}$ de longitude, 
fazendo parte do sistema da serra da Mantiqueira. Estende-se mais ou menos no sentido leste-oeste por cerca de $12 \mathrm{Km}$, percorrendo terras dos municípios de São João del Rei, Tiradentes, Coronel Xavier Chaves e Prados. Trata-se de um maciço de arenito quartzítico que ergue-se a uma altitude de cerca de $1400 \mathrm{~m}$, destacando-se da paisagem circundante, onde predominam altitudes de cerca $900 \mathrm{~m}$. O topo da serra, localmente chamado de 'mangue', é revestido por campo rupestre. $\mathrm{Na}$ face norte, a serra perde altitude mais gradualmente que na face sul, onde escarpas abruptas assentam-se sobre contrafortes bastante inclinados os quais alojam as nascentes de vários cursos d'água que fluem para 0 sul, até o rio das Mortes. Estes contrafortes são cobertos por uma floresta estacional semidecícua montana (terminologia segundo Veloso et al., 1991), a qual foi objeto do presente estudo. $O$ tipo de solo predominante é o Cambissolo álico textura arenosa (dados inéditos dos autores). $\mathrm{O}$ clima da região é do tipo $\mathrm{Cwb}$, ou seja, mesotérmico com estações bem definidas: uma fria e seca, entre abril e setembro, e uma quente e chuvosa, entre outubro e maio (Carvalho, 1987).

O levantamento florístico foi realizado durante o ano de 1991 a partir de coletas de material botânico feitas na floresta por técnicos da EFLEX e por professores e alunos da ESAL. O material coletado pela EFLEX, em particular, foi obtido em 95 parcelas de $10 \mathrm{x}$ $10 \mathrm{~m}$ demarcadas no interior da floresta com o propósito de realizar um levantamento fitossociológico. Foi coletado material botânico de todas as espécies cujos indivíduos apresentassem uma circunferência à altura do solo, CAS, $>=5 \mathrm{~cm}$. As coletas feitas pela ESAL concentraram-se ao longo do córrego Mãe d'Água. A identificação do material botânico foi realizada com auxílio de literatura pertinente e de consultas aos especialistas e às coleções dos herbários do Jardim Botânico do Rio de Janeiro (RB), Instituto de Botânica de São Paulo (SP) e Universidade Estadual de Campinas (UEC). Os espécimes coletados foram depositados no Herbário do Departamento de Biologia da ESAL.

Foram realizadas comparações florísticas quantitativas com outros levantamentos realizados em florestas de altitude nos estados de São Paulo e Minas Gerais. Foi utilizada a altitude mínima de $750 \mathrm{~m}$ como limite, uma vez que Torres et al. (1993) demonstraram que este valor foi eficiente em discriminar floristicamente as florestas de altitude no estado de São Paulo. A medida de similaridade adotada foi o índice de Jaccard (Brower \& Zar, 1984). Os levantamentos foram selecionados de acordo com os seguintes critérios: (a) apresentarem um razoável número de espécies de árvores e arbustos na listagem (mínimo de 150), (b) apresentarem mais de $80 \%$ de identificações a nível de espécie, (c) distingüirem claramente a ocorrência das espécies no tipo fisionômico florestal. Estes critérios foram estabelecidos para se obter um mínimo de homogeneidade entre os levantamentos, necessário para permitir comparações consistentes.

\section{Resultados e discussão}

Os resultados do levantamento florístico encontram-se na Tabela 1. Foram registradas 277 espécies, das quais três são pteridófitas (duas famílias: Blechnaceae e Cyatheaceae), uma é gimnosperma (família Podocarpaceae) e as 273 restantes são angiospermas, representadas por 64 famílias, sendo apenas uma delas de monocotiledôneas, Arecaceae, com três espécies. A Figura 1 representa as famílias ordenadas pelo número de espécies 
Tabela 1 - Relação das espécies de árvores e arbustos encontradas na floresta semidecídua montana da Serra de São José, Tiradentes, MG. As espécies encontram-se seguidas de seus nomes vernaculares e do númerode registro do espécime depositado no Herbário da ESAL (sub-coleção Convênio CEMIG/ESAL/FAEPE).

Anacardiaceae:

Lithraea molleoides (Vell.) Engler

Schinus terebinthifolius Raddi

Tapirira guianensis Aublet

Tapirira obtusa (Benth.) Mitchell aroeira-branca, aroeirinha

aroeira-fria, aroeira-vermelha

fruta-de-pombo, pombeiro

pombeiro-branco, pau-pombo
12332

12786

12331

07784

Annonaceae:

Annona cacans Warm.

Guatteria nigrescens Mart.

Guatteria vilosissima A.St.-Hil.

Rollinia laurifolia Schlecht

Rollinia sericea R.E. Fries

Rollinia sylvatica Mart.

Xylopia brasiliensis Sprengel

Xylopia sericea A.St.-Hil.

Apocynaceae:

Aspidosperma cylindrocarpon

Müll. Arg.

peroba-poca

13164

Aspidosperma parvifolium A. DC.

guatambu, pereiro

12314

Aspidosperma polyneuron Müll. Arg.

cabo-de-machado, peroba

13032

Aspidosperma ramiflorum Müll. Arg.

guatambu, peroba-rosa

12313

Aquifoliaceae:

Ilex cerasifolia Reisseck

congonha-peluda

12799

Ilex conocarpa Reisseck

catuaba-do-mato, congonha

13096

Ilex dumosa Reisseck

congonha-tostão

13097

Araliaceae:

Dendropanax cuneatum (DC.)

Decne \& Planchon

mandioca, maria-mole

12310

Didymopanax angustissima

E. Marchand

mandiocão, morototó

12798

Arecaceae:

Arecastrum romanzoffianum

(Cham.) Becc.

coco-baboso

14055

Geonoma schottiana Mart.

guaricanga, aricanga

12537

Syagrus oleracea (Mart.) Becc.

coco-catolé 
Asteraceae:

Baccharis dentata (Vell.)

G. M. Barroso

alecrim-de-árvore $\quad 12986$

Eremanthus incanus Less.

candeinha, pau-de-candeia $\quad 09596$

Gochnatia polymorpha (Less.) Cabrera

Piptocarpha axillaris (Less.) Baker

camará, candeia-podre

02612

Piptocarpha rotundifolia (Less.) Baker

vassoura-preta, canela-podre

12803

Vanillosmopsis erythropappa Schultz

cambará-do-campo

02588

Vernonia diffusa Less.

Vernonia discolor Less

candeia-verdadeira, cambará

13232

vassourão-preto

12982

vassourão-preto, cambará

12800

Bignoniaceae:

Jacaranda macrantha Cham.

caroba-do-mato

12353

Sparattosperma leucanthum

(Vell) K.Schum.

Tabebuia alba (Cham.) Sandw.

ipê-cabeludo

13094

ipê-da-serra 11318

Tabebuia ochracea (Cham.) Rizz.

ipê-cascudo, piúna-do-campo

03114

Tabebuia roseo-alba (Ridl.) Sandw.

ipê-branco, peroba

05663

Tabebuia serratifolia (Valh.) Nichols

ipê-amarelo

03115

Blechnaceae:

Blechnum brasiliense Desv.

samambaia-do-brejo

13217

Bombacaceae:

Ceiba speciosa (A.St.-Hil.)

Gibbs \& Semir.

Pseudobombax longiflorum

(Mart. \& Zucc) A. Robyns

paineira

13092

paineira-lisa, imbiruçu

03309

Boraginaceae:

Cordia rufescens A.DC.

Cordia sellowiana Cham.

Cordia superba Cham.

mulato-branco, bago-de-boi

12294

chá-de-bugre, juruté $\quad 12348$

grão-de-galo

13010

Burseraceae:

Protium almecega Marchand

almecega-de-casca-lisa, breu

12345

Protium brasiliense (Sprengel) Engler

almecegueira, breu

Protium heptaphyllum (Aublet)

Marchand

breu-vermelho

12346

Protium widgrenii Engler

almecega-cascuda

09416

Caesalpiniaceae:

Cassia ferruginea (Schrad.) ex DC. canafistula, fedegoso 
Copaifera langsdorffii Desf.

Hymenaea courbaril L.

Sclerolobium rugosum Mart. form.

plurijugum Mell. Barr.

Senna macranthera (Vell.)

Irwin \& Barneby

Cecropiaceae:

Cecropia glazioui Snethl.

Cecropia hololeuca Miq.

Cecropia pachystachya Trécul

Celastraceae:

Austroplenckia populnea

(Reisseck) Lund.

Maytenus aquifolium Mart.

Maytenus gonoclados Mart.

Chloranthaceae:

Hedyosmum brasiliense Mart.

Chrysobalanaceae:

Hirtella glandulosa Sprengel

Licania hoehnei Pilger

Clethraceae:

Clethra scabra Pers.

Clusiaceae:

Calophyllum brasiliense Cambess.

Clusia criuva Cambess.

Kielmeyera lathrophyton Saddi

Rheedia gardneriana

Planchon \& Triana

Tovomitopsis saldanhae Engler

Vismia brasiliensis Choisy

Combretaceae:

Terminalia glabrescens Mart.

Connaraceae:

Connarus regnellii Schelemberg copaíba-vermelha, pau-d'óleo

12448

jabotá-d'anta, jataí

12446

angá, cangalheiro, carvoeiro

12859

fedegoso, amarelinho

09403

embaúba-vermelha

14003

embaúba-branca

14021

embaúba-cinzenta

12035

carvalho-do-campo

00127

espinheira-santa

13031

congonha

13233

espirradeira-da-mata

13029

azeitona

05728

ticum

13089

vermelho, canjuja, vassourão

guanand, mangue

12384

estalo, criúva

13084

pau-santo

13104

bacupari-miúdo, bacoparí

12383

azedinho, juruvoca

12382

pau-de-lacre, purga-de-vento

12379

carvalho, mirindiba

12338 
Cunoniaceae:

Lamanonia ternata Vell. cedrilho

Cyatheaceae:

Cyathea delgadii Sternb.

samambaiaçu-vermelho

12333

Nephelea sternbergii (Sternb.) Tryon

samambaiaçu-branco

12810

Elaeocarpaceae:

Sloanea monosperma Vell. ouriço, castanha-brava

12372

Erythroxylaceae:

Erythroxylum campestre A.St.-Hil. fruta-de-tucano, garibaldi $\quad 12369$

$\begin{array}{lll}\text { Erythroxylum citrifolium A.St.-HHil. fruta-de-juriti } & 12808\end{array}$

Euphorbiaceae:

Actinostemon communis

(Müll. Arg.) Pax laranjeira-brava

12365

Alchornea glandulosa Poepp. \& Endl. tapiá, tanheiro

Alchornea triplinervea

(Sprengel) Müll. Arg.

tanheiro, tapiá-vermelho

12362

Aparisthmium cordatum (Juss.) Baill.

pau-de-facho

13222

Croton echinocarpus Müll. Art.

sangra d'água, sangue-de-drago

13384

Croton floribundus Sprengel

Croton urucurana Baill.

Drypetes sessiliflora $\mathrm{Fr}$. Allem.

tapichingui, capichingui

12358

sangra d'água, sangue-de-drago 12189

folha-de-serra-grande

12840

Hieronyma ferruginea Tul.

Mabea fistulifera Mart.

Pera obovata Baill.

Sebastiania serrata Müll. Arg.

sangue-de-boi, quina-do-pará

12356

canudo-de-pito

12655

pau-de-sapateiro, cacho-de-arroz 12354

esporão-de-espinho, branquinho 13085

Fabaceae:

Acosmium dasycarpum

(Vogel) Yakovlev

sucupira-da-serra, chapada

12661

Acosmium subelegans

(Vogel) Yakovlev

sucupira-do-campo

Andira anthelmia

(Vell.) Macbr.

Bowdichia virgilioides Kunth

Dalbergia brasiliensis Vogel

Dalbergia miscolobium Benth.

Dalbergia villosa (Benth.) Benth.

Machaerium aculeatum Raddi

Machaerium nictitans Bent.

angelim-amargo, pau-de-morcego 12994

sucupira-preta

12858

jacarandá

14002

cabiúna-do-campo

05508

milho-torrado

12437

adolfo, jacarandá-de-espinho

jacarandá-ferro, bico-de-pato

12430 
Machaerium scleroxylum Tul.

Machaerium stipitatum (DC.) Vogel

Machaerium villosum Vogel

Platycyamus regnellii Benth.

Platypodium elegans Vogel

Flacourtiaceae:

Casearia decandra Jacquin

Casearia gossypiosperma Briquet

Casearia lasiophylla Eichler

Casearia sylvestris Sw.

Hippocrateaceae:

Salacia elliptica

(Mart. ex Schul.) E.Don.

Lacistemaceae:

Lacistema hasslerianum Chodat

Lamiaceae:

Hyptis arborea Benth.

Lauraceae:

Beilschmiedia emarginata

(Meissner) Kosterm

Cryptocarya aschersoniana $\mathrm{Mez}$

Endlicheria paniculata

(Sprengel) Macbr.

Nectandra cissiflora Nees

Nectandra nitidula Nees \&

Mart. ex Nees

Nectandra oppositifolia Nees

Nectandra puberula (Schott) Nees

Nectandra reticulata $\mathrm{Mez}$

Nectandra warmingii Meissner

Ocotea acutifolia (Nees) Mez

Ocotea corymbosa (Meissner) Mez

Ocotea odorifera (Vell.) Rohwer

Ocotea pulchella (Nees) Mez

Persea pyrifolia Nees \& Mart. ex Nees cabiúna

13229

jacarandá-roxo, sapuva, ximbó

12427

jacarandá-mineiro

12426

pau-pereira, cataguá

12423

jacarandá-branco, faveiro

12115

canela-espeto, cambroé

12397

japeiro, língua-de-tiú

13230

espeto-peludo

12201

erva-de-lagarto, guaçatonga

12203

bacupari

12376

cafeeiro-do-mato

02696

salva-do-campo

13125

canela-ameixa

12848

canela-branca, canela-de-jacu

12417

canela-peluda, canela-do-brejo

12416

canelão

13026

canela-amarela

12414

canela-amarela, canela-ferrugem 12411

louro-amarelo, fruta-de-jacu 13081

canela-amarela

12966

canela-amarela

12214

canelinha-da-serra

13024

canela-bosta, canela-preta

12406

canela-sassafrás, sassafrás

12407

canela-prego, canelinha

12993

massaranduba, nicurana

Lecythidaceae:

Cariniana legalis (Mart.) Kuntze

jequitibá-rosa

14009 
Loganiaceae:

Strychnos brasiliensis Mart.

solta-martinho

12454

Lythraceae:

Lafoensia pacari A.St.-Hil. dedaleira

13103

Magnoliaceae:

Talauma ovata A.St.-Hil. pinha-do-brejo, baguaçu

10193

Malpighiaceae:

Byrsonima intermedia A.Juss. murici-miúdo, canjica 12888

$\begin{array}{lll}\text { Byrsonima laxiflora Gris. } & \text { murici-da-mata }\end{array}$

Melastomataceae:

$\begin{array}{lll}\text { Leandra scabra DC. } & \text { pixirica, camará-do-mato } & 12239\end{array}$

Miconia albicans Triana canela-de-velho, olhos-de-porco 12883

Miconia argyrophylla DC. casca-de-arroz 12494

Miconia brunnea Mart. ex DC. cabuçu, carvão-folha-larga 13077

Miconia chartacea Triana mexeriquinha 12493

Miconia cinnamomifolia (DC.) Naudin voadeira, jacatirão 12880

Miconia corallina Sprengel carvãozinho 09640

Miconia cubatanensis Hoehne carvãozinho 12666

$\begin{array}{lll}\text { Miconia hispida } \text { Cogn. } & 12491\end{array}$

Miconia minutiflora (Bonph.) Triana pedra-uni, jacatirão 13078

Miconia pepericarpa DC. $\quad$ carvãozinho-vermelho 12236

$\begin{array}{lll}\text { Miconia rigidiuscula } \text { Cogn. } \quad \text { pixirica, carvãozinho-branco } & 12877\end{array}$

Miconia tristis Sprengel jacatirão 12490

Mouriri glazioviana Cogn. mandapuçá 13336

Tibouchina candolleana (DC.) Cogn. quaresma 12238

Tibouchina stenocarpa (DC.) Cogn. quaresmão, cuiupeva 12876

Trembleya parviflora (Don) Cogn. flor-de-quaresma 12235

Meliaceae:

$\begin{array}{lll}\text { Cabralea canjerana (Vell) Mart. } \quad \text { cangerana, pindaiborana } & 09627\end{array}$

Cedrela fissilis Vell. cedro-rosa, cedro-branco 12234

Guarea guidonia (L.) Sleumer cura-madre, marinheiro 12233

Guarea macrophylla Vahl. cura-madre, marinheiro 13019

Mimosaceae:

Anadenanthera colubrina

(Vell.) Brenan angico

13242

Anadenanthera peregrina

(Benth.) Speg.

angico-vermelho 
Inga affinis DC.

ingá-doce, ingá-miúdo

09513

Inga luschnatiana Benth.

ingaçu

Inga striata Benth.

ingá

13241

Inga uruguensis Hook. \& Arn.

ingá

12866

Piptadenia gonoacantha

(Mart.) Macbr.

pau-jacaré, monjoleiro

12865

Pithecellobium incuriale

(Vell.) Benth.

Pseudopiptadenia leptostachya

(Benth.) Rausch.

cortiço, angio-do-campo

12445

Stryphnodendron polyphyllum Mart.

itapicuru

12863

barbatimão

13021

Monimiaceae:

Mollinedia argyrogyna Perk.

congonha-branca

12477

Mollinedia triflora (Sprengel) Tul.

capixim, pau-de-espeto

12689

Mollinedia uleana Perk.

cafeeiro-do-mato

13060

Siparuna apiosyce (Mart.) DC.

Siparuna guianensis Aublet

limoeiro-bravo, folha-santa

12473

negramina, capitiú

12471

Moraceae:

Ficus luschnatiana (Miq.)Miq..

figueira

13061

Ficus mexiae Standl.

figueira-preta

12465

Ficus pertusa L.f.

figueira, pau-de-corda

13018

Sorocea bonplandii

(Baillon) W. Burger

canxim, cincho

12463

Myrsinaceae:

Myrsine coriacea (Sw.) R.Br.

pororoca-branca

12227

Myrsine guianensis (Aublet) Kuntze

pororoca-cascuda

02779

Myrsine lancifolia Mart.

pororoquinha-branca

12894

Myrsine oblonga (Pohl.) Pipoly

pororoca-vermelha

14007

Myrsine umbellata Mart.

pororoca-branca

12460

Stylogyne ambigua (Mart.) Mez

pororoquinha-vermelha

12274

Myrtaceae:

Calycorectes acutatus (Miq.) Toledo

amarelinho, grumixama

12250

Calyptranthes clusiaefolia (Miq.) Berg

jaborandi

12524

Calyptranthes lucida Mart. ex DC.

jambo-do-mato, batinga-magra

12510

Calyptranthes strigipes Berg

ruão

09639

Campomanesia guazumifolia

(Cambess.) Berg

sete-capotes

12523

Campomanesia pubescens (DC.) Berg

guabiroba

13228

Eugenia aurata Berg

murtinha, canzil

12926 
Eugenia blastantha (Berg) Legr. Eugenia involucrata DC.

Eugenia myrtifolia Cambess.

Eugenia pluriflora DC.

Eugenia uniflora $\mathrm{L}$.

Gomidesia affinis (Cambess.) Legr.

Gomidesia eriocalyx (DC.) Legr.

Gomidesia lindeniana Berg

Myrcia multiflora (Lam.) DC.

Myrcia rostrata DC.

Myrcia rufipes DC.

Myrcia iomentosa (Aublet) DC.

Myrcia velutina Berg

Myrcia venulosa DC.

Myrciaria floribunda

(West ex Willd.) Berg

Pimenta pseudocaryophyllus

(Gomes) Landrum

Psidium guineense Sw.

Psidium rufum Mart.

Siphoneugena densiflora Berg

Siphoneugena widgreniana Berg

Syzygium jambos (L.) Alston casca-de-papel, guamirim

12924

pitanga-preta

12682

pitanguinha-preta

olho-de-boi

12515

12526

pitanga-vermelha

09528

guamirim

12518

guamirim-orelhinha

09612

guamirim-de-folha-grande

13133

cambui

12699

guamirim-de-folha-miúda

12262

guamirim-lagoa

12253

goiabão, goiabeira-brava

12260

piúna, folha-miúda-cascuda

09653

guamirim-do-campo

12517

jaboticabinha

13016

craveiro-da-terra, cravo

12933

araçá-preto, araçá-perinha

13002

araçá-do-campo

13015

cravinho, piúna

12519

cravinho, lagoa-miúda

12939

jambo-paubeiro

Nyctagomaceae:

Pisonia ambigua Heimerl

joão-mole

14006

Olacaceae:

Heisteria silvianii Schwarke umari

13221

Opiliaceae:

Agonandra engleri Hoehne

cerveja-de-pobre, estoque

12540

Fiperaceae:

Ottonia leptostachya Kunth

jaborandi, jaguarundi

12268

Piper aduncum L.

Piper caracolanum C.DC.

erva-de-jaboti, aperta-mão

12271

fruto-de-morcego

12535

Piper gaudichaudianum Kunth

Pothomorphe umbellata (L.) Miq.

caapeba

09763

caapeba-do-norte

12270

Podocarpaceae:

Podocarpus lamberiii Klotzsch

pinheirinho 
Proteaceae:

Euplassa incana (Klotzsch)

Johnston Sleumer

cabatã, catinga-de-barrão

12582

Euplassa rufa (Loesen) Sleumer Engler

catinga-de-barrão

12583

Roupala brasiliensis Klotzsch

carne-de-vaca, catucaém

12533

Roupala longepetiolata Pohl

carne-de-vaca, carvalho

13034

Roupala montana Aublet

carne-de-vaca

02686

Rhamnaceae:

Colubrina glandulosa Perkins

sobrasil, saguaraji-vermelho

13035

Frangula polymorpha Reisseck

saguaraji-vermelho

13036

Rhamnidium elaeocarpum Reisseck

saguaraji-amarelo, tarumaí

12671

Rosaceae:

Prunus sellowii Koehne

pessegueiro-bravo

12573

Rubiaceae:

Alibertia concolor Schum.

marmelada

05743

Alibertia macrophylla Schum.

marmelada-de-cachorro

12672

Alibertia sessilis (Vell.) Schum.

marmelinho-do-campo

01748

Amaioua guianensis Aublet

Chomelia sericea Müll. Arg.

marmelada, canela-de-veado

12560

veludo-branco

12559

Coutarea hexandra (Jacq.) Schum.

veludo-preto

12557

Faramea cyanea Müll. Arg.

cafezinho

09718

Faramea multiflora A. Rich. ex DC.

café-do-mato

12553

Guaitarda viburnioides (Cham.) Schltr.

Ixora warmingii Müll. Arg.

jangada, angélica

12278

ixora-do-mato

12552

Psychotria carthagenensis Jacquin

Psychotria hastisepala Müll. Arg.

pau-de-maria, guamirim-preto

12285

Psychotria sessilis (Vell.) Müll. Arg.

pau-de-espeto, araçá-bravo

12280

Randia nitida (Kunth.) DC.

folha-miúda, azedeira

12282

limão-bravo

12277

Rudgea viburnioides (Cham.) Benth.

congonha-de-bugre

12957

Rutaceae:

Angostura pentandra

(A.St.-Hil.) Albuquerque

angostura

14011

Esenbeckia febrifuga

(A.St.-Hil.) A.Juss.

mamoninha, guaxupita

12571

Galipea multiflora Schult.

guamixinga, grumarim

12673

Metrodorea nigra A.St.-Hil.

arco-de-pipa, caputuna

14012

Zanthoxylum rhoifolium Lam.

mamica-de-porca

12565

Sabiaceae:

Meliosma sellowii Urban

congonha-de-folha-larga 
Sapindaceae:

Cupania oblongifolia Mart.

Cupania vernalis Cambess.

Dilodendron bipinnatum Radlk.

Matayba elaeagnoides Radlk.

Matayba guianensis Aublet

Matayba juglandifolia

(Cambess.) Radlk.

cabatã, camboatã $\quad 14015$

pau-de-cantil, gragoatã $\quad 12297$

puta-pobre, mulher-pobre $\quad 13064$

caqui-do-mato, cragoat-branco 12298

cragoatã

12587

caqui-do-mato, cragoatã-branco 12586

Sapotaceae:

Chrysophyllum marginatum

(Hook \& Arn.) Radlk.

gumbijava, guatambu-de-leite

Solanaceae:

Brunfelsia brasiliensis

(Sprengel) Smith \& Downs

manacá

09615

Cestrum laevigatum Schltr.

Solanum erianthum Don

Solanum granuloso-leprosum Dun

dama-da-noite, coeirana

12696

jurubeba-de-árvore

13039

jurubeba-de-árvore

13040

Solanum inaequale Vell.

Solanum martii Sendt.

fedorento, cuivira, coeirana

12967

braço-de-mono, marianeira

13041

Sterculiaceae:

Guazuma ulmifolia L.

pau-de-motamba, camacã

12578

Styracaceae:

Styrax camporum Pohl

sabão, benjoim, canelão

12291

Styrax pohlii A.DC.

benjoeiro, salgueiro

12576

Symplocaceae:

$\begin{array}{lll}\text { Symplocos celastrinea Mart. ex Miq. congonha } & 14017\end{array}$

Symplocos lanceolata (Mart.) A.DC. congonha 13043

Symplocos pubescens Klotzsch sete-sangrias, saboeiro

13213

Theaceae:

Laplacea semiserrata Cambess. mangue 13073

Ternstroemia alnifolia Wawra pororoca-vermelha 13072

Thymelaeaceae:

Daphnopsis brasiliensis Mart. \& Zucc. imbira-branca, imbira-de-sapo 12596

Daphnopsis fasciculata (Meissner) Nevl. imbira-vermelha 09795

Tiliaceae:

Luehea divaricata Mart. açoita-cavalo

Luehea grandiflora Mart. \& Zucc. açoita-cavalo 
Ulmaceae:

Celtis iguanea (Jacq.) Sarg.

Trema micrantha Blume grão-de-galo, joá-miúdo

crindiúva, pau-de-pólvora
12301

12302

\section{Verbenaceae:}

Aegiphila lhotzkiana Cham.

pau-de-papagaio

12300

Aegiphila sellowiana Cham.

briaúva, pau-de-tamanco

12677

Aloysia virgata A.Juss.

pau-lixa

04921

Lantana fucata Lindl.

alecrim-de-árvore

13007

Vitex polygama Cham.

maria-preta, velame-do-campo

13009

Vochysiaceae:

Qualea multiflora Mart.

bagre, pau-terra, cinzeiro

12299

Vochysia tucanorum Mart.

registradas. As famílias com os maiores valores, Myrtaceae, Melastomataceae, Rubiaceae, Fagaceae, Lauraceae e Euphorbiaceae, estão igualmente entre as que apresentam o maior número de espécies na maioria dos levantamentos florísticos realizados em florestas semidecíduas (matas de planalto) do estado de São Paulo (Leitão Filho, 1987).

No perfil florístico da presente listagem destaca-se ainda um significativo número de espécies características de florestas montanas, ou de altitude. De uma listagem de 41 espécies de árvores e arbustos indicadoras de florestas de altitude do sul-sudeste do Brasil produzida por Meira Neto et al. (1989), 35 (85,3\%) constam também na presente listagem. Além destas, certamente poderiam ser acrescentadas outras mais, características (mas não exclusivas) das florestas montanas do sistema da Mantiqueira, como Clethra scabra, Eremanthus incanus, Vanillosmopsis erythropappa, Vismia brasiliensis, Hieronyma ferruginea, Roupala longepetiolata, Protium widgrenii, Euplassa rufa, Sclerolobium rugosum e Podocarpus sellowii (Carvalho et al., 1992; Gavilanes et al., 1992a, 1992b; Oliveira-Filho et al., 1993; Vilela et al., 1993).

Poucos levantamentos realizados no estado de Minas Gerais atenderam aos critérios estabelecidos para se fazerem comparações consistentes. O motivo básico para esta dificuldade é que a maioria dos trabalhos não utilizados é constituída de valiosos levantamentos exploratórios que incluem espécies de todos os estratos de diversas fisionomias da vegetação (e.g. Brandão, 1992; Brandão \& Gavilanes, 1990; Ferreira \& Magalhães, 1977; Ferreira et al., 1977/78). Desta forma, o número de espécies arbustivoarbóreas de fomações florestais nestes levantamentos resultou relativamente diluído. Por outro lado, todos os estudos aproveitados nas comparações, sejam eles de Minas Gerais ou de São Paulo, provêm de levantamentos destinados a subsidiar estudos fitossociológicos. Portanto, é perfeitamente compreensível que estudos desta natureza resultem em listagens maiores, uma vez que o levantamento fitossociológico exige o exame de cada indivíduo no interior de uma área amostral. Além disso, as visitas ao campo são, em geral, rotineiras e se estendem ao longo de pelo menos um ano. 


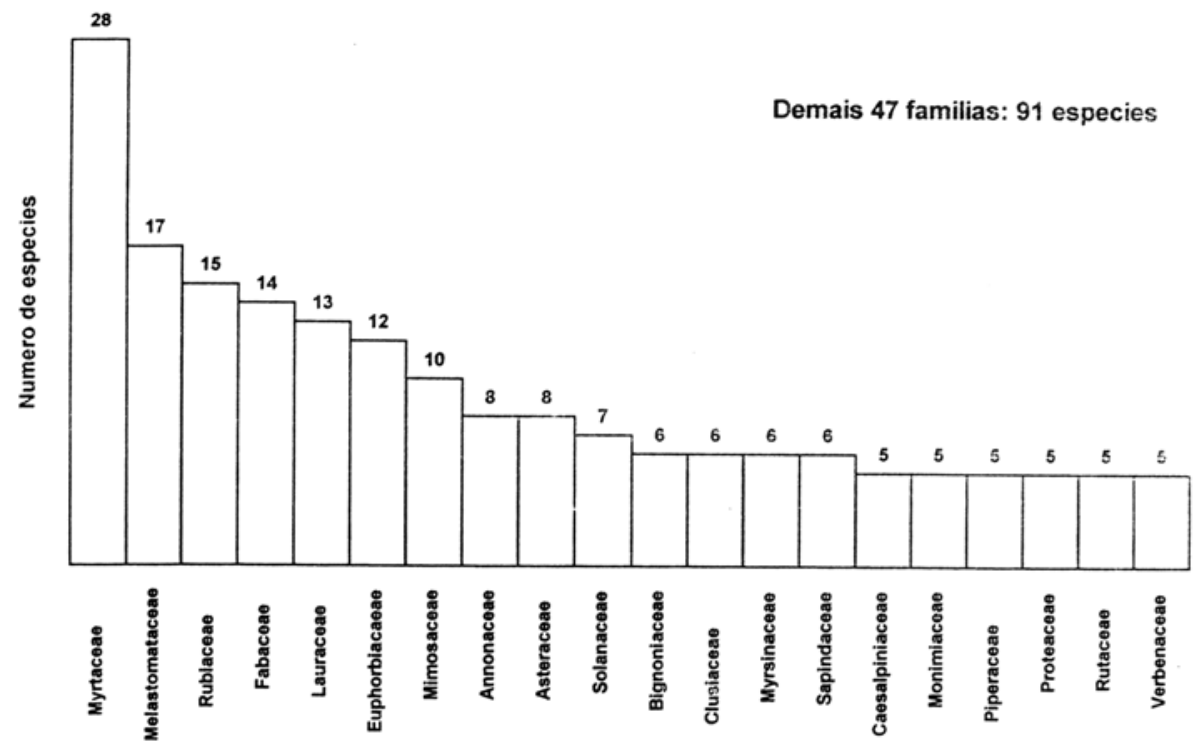

Figura 1 - Distribuição do número de espécies por famílias na floresta semidecídua montana da serra de São José, Tiradentes, MG.

Os resultados das comparações com outras florestas de altitude do Sudeste do Brasil encontram-se na Tabela 2. Como se pode observar, os maiores valores (Is $>30 \%$ ) foram obtidos para levantamentos realizados no sul de Minas Gerais, na região do Alto Rio Grande (munićpios de Lavras, Bom Sucesso, Itutinga e Madre de Deus de Minas). Esta maior similaridade deve-se, com certeza à maior proximidade geográfica, na medida em que esta significa maiores semelhanças ambientais, sobretudo climáticas. A região sul de Minas Gerais compreende a vertente continental do sistema da Mantiqueira, onde a vegetação primitiva compreendia um complexo mosaico entre floresta ombrófila mista, floresta semidecídua montana, campos rupestres, campos limpos de altitude e crrado (Carvalho, 1987; IBGE, 1984). De acordo com Eiten (1982), este mosaico vegetacional do sul de Minas Gerais deve-se à combinação de dois fatores: a transição para os climas 
Tabela 2 - Índice de similaridade florística de Jaccard, Is, calculados entre o presente levantamento e outras listagens de espécies arbustivo-arbóreas produzidas para florestas semidecíduas montanas do Sudeste Brasileiro. $\mathrm{N}$ = Número de espécies arbustivo-arbóreas na listagem; $\mathrm{c}=$ número de espécies em comum com o presente trabalho. Referências ordenadas por valores decrescentes de Is.

\begin{tabular}{|c|c|c|c|c|c|}
\hline Local & Referência & $\begin{array}{l}\text { Altitude } \\
\text { média } \\
(\mathrm{m})\end{array}$ & $\mathrm{N}$ & c & Is $(\%)$ \\
\hline $\begin{array}{l}\text { Represa de Camargos, } \\
\text { Itutinga, MG }\end{array}$ & Vilela et al., 1993 & 917 & 256 & 181 & 51.42 \\
\hline $\begin{array}{l}\text { Campus da ESAL, } \\
\text { Lavras, MG }\end{array}$ & Oliveira-Filho et al., 1993 & 925 & 184 & 138 & 42.72 \\
\hline $\begin{array}{l}\text { Reserva do Poço Bonito, } \\
\text { Lavras, MG }\end{array}$ & Gavilanes et al., 1992a & 1150 & 219 & 147 & 42.12 \\
\hline Madre de Deus de Minas, MG & Gavilanes et al., 1992b & 925 & 189 & 120 & 34.68 \\
\hline Bom Sucesso, MG & Carvalho et al., 1992 & 825 & 217 & 123 & 33.15 \\
\hline $\begin{array}{l}\text { Parque da Grota Funda, } \\
\text { Atibaia, SP }\end{array}$ & Meira Neto et al., 1989 & 1250 & 201 & 91 & 23.51 \\
\hline Est. Ecológica de Angatuba, SP & Torres, 1989 & 900 & 176 & 86 & 23.43 \\
\hline Serra do Japi, Jundiai, SP & Rodrigues, 1986 & 1020 & 207 & 91 & 23.15 \\
\hline Aeroporto Intern., Guarulhos, SP & Gandolfi, 1991* & 742 & 158 & 81 & 22.88 \\
\hline São José dos Campos, SP & Silva, 1989 & 840 & 195 & 74 & 18.59 \\
\hline Inst. Botânica, São Paulo, SP & De Vuono, 1985 & 798 & 182 & 41 & 9.81 \\
\hline
\end{tabular}

* Este estudo foi incluído, apesar de situar-se abaixo da altitude limite de $750 \mathrm{~m}$, devido à pequena diferença de apenas $8 \mathrm{~m}$.

mais fortemente estacionais do Brasil Central e as altitudes elevadas do sistema da Mantiqueira. Estas particularidades certamente contribuem para uma maior similaridade entre as florestas da região.

Entre os cinco levantamentos realizados na região do Alto Rio Grande os maiores valores de similaridade foram obtidos para Itutinga, Lavras/ESAL e Lavras/Poço Bonito e os menores para Madre de Deus de Minas e Bom Sucesso. Isto deve-se, provavelmente, ao fato de estas duas últimas áreas localizarem-se às margens do Rio Grande e, portanto, 
incluírem dois tipos de floresta semidecídua: aluvial e montana (sensu Veloso et al., 1991). As demais áreas, como a do presente estudo, encontram-se em sítios elevados, distantes dos grandes rios, compreendendo apenas florestas semidecíduas montanas. Em relação às florestas de altitude do estado de São Paulo, os maiores valores de similaridade (todos muito próximos) foram encontrados para Atibaia, Jundiaí e Angatuba, áreas que correspondem também aos maiores valores de altitude média, entre 900 e $1250 \mathrm{~m}$. A maior altitude nestas áreas possivelmente explica sua maior similaridade florística com a floresta da serra de São José a qual estende-se também entre os 900 e $1100 \mathrm{~m}$ de altitude.

Outro importante aspecto a ser salientado na presente listagem é a notável riqueza de espécies, que pode ser evidenciada quando se compara o total de 277 espécies de árvores e arbustos registradas para a serra de São José com outros valores obtidos em levantamentos realizados em florestas da região com métodos e intensidades eqüivalentes: $256 \mathrm{em}$ Itutinga (Vilela et al., 1993), $217 \mathrm{em}$ Bom Sucesso (Carvalho et al., 1992), 189 em Madre de Deus de Minas (Gavilanes et al., 1992b) e 219 em Lavras (Oliveira-Filho et al., 1993). Esta notável riqueza constitui um recurso natural extremamente valioso e, portanto, sua conservação é uma medida justificável não apenas pela sua importância sócio-econômica para a comunidade local, mas também pelo patrimônio genético que encerra.

\section{Referências Bibliográficas}

Brandão, M. 1992. Caracterização geomorfológica, climática, florística e faunística da Serra do Curral em Belo Horizonte, MG. Daphne 2:5-12.

Brandão, M. \& Gavilanes, M.L. 1990. Mais uma contribuição para o conhecimento da Cadeia do Espinhaço em Minas Gerais (Serra da Piedade) - II. Daphne 1:26-43.

Brower, J.E. \& Zar, J.H. (1984). Field and Laboratory Methods for General Ecology. 226 pp., Dubuque, Wm. C. Brown Pub..

Carvalho, D.A. 1987. Composição florística e estrutura de cerrados do sudoeste de Minas Gerais. Campinas, Universidade Estadual de Campinas, tese de doutoramento.

Carvalho, D.A., Oliveira-Filho, A. T., Vilela, E. A. \& Gavilanes, M.L. 1992. Flora arbustivo-arbórea das matas ciliares do Alto Rio Grande (MG). 1 - mata de Macaia (Bom Sucesso). In: Anais do $2^{\circ}$ Congresso Nacional sobre Essências Nativas, São Paulo, 1992. pp. 274-282.

De Vuono, Y.S. 1985. Fitossociologia do estrato arbóreo da floresta da Reserva Biológica do Instituto de Botânica (São Paulo, SP). São Paulo, Universidade de São Paulo, tese de doutoramento.

IBGE. 1984. Mapa de vegetação do Brasil. Instituto Brasileiro de Geografia e Estatística (IBGE), Rio de Janeiro.

Eiten, G. 1982. Brazilian "Savannas", p. 25-47 in Huntley, B.J. \& Walker, B.H. (eds.) Ecology of Tropical Savannas. Berlim, Springer-Verlag.

Ferreira, M.B., D’Assumpção, W. R. C. \& Magalhães, G. M. 1977/78. Nova contribuição para o conhecimento da vegetação da Cadeia do Espinhaço ou Serra geral (Maciço do Caraça). Oreades 6:49-67. 
Ferreira, M.B. \& Magalhães, G.M. 1977. Mais uma contribuição ao conhecimento da vegetação da Serra do Espinhaço em Minas Gerais; I. Serra do Grão Mogol e de Ibitipoca. In: Anais do XXVI Congresso Nacional de Botânica, Rio de Janeiro, 1975, Academia Brasileira de Ciências. p. 175-183.

Gandolfi, S. 1991. Estudos florístico e fitossociológico de uma floresta residual na área do Aeroporto Internacional de São Paulo, município de Guarulhos, SP. Campinas, Universidade Estadual de Campinas, dissertação de mestrado.

Gavilanes, M. L., Brandão, M., Oliveira-Filho, A. T., Almeida, R.J., Mello, J.M. \& Avezum, F.F. 1992a. Flórula da Reserva Biológica Municipal do Poço Bonito, Lavras, MG. III - formação florestal. Daphne 2:14-26.

Gavilanes, M.L., Oliveira-Filho, A.T., Carvalho, D.A. \& Vilela, E.A. 1992b. Flora arbustivo-arbórea de uma mata ciliar do Alto Rio Grande, em Madre de Deus de Minas - MG. Daphne 2:15-24.

Leitão Filho, H.F. 1987. Considerações sobre a florística de florestas tropicais e subtropicais do Brasil. IPEF 35:41-46.

Meira Neto, J.A.A., Bernacci, L.C., Grombone, M.T., Tamashiro, J.Y. \& Leitão Filho, H.F. 1989. Composição florística da floresta semidecídua de altitude do Parque Municipal da Grota Funda (Atibaia, estado de São Paulo). Acta bot. bras. 3:51-74.

Oliveira-Filho, A.T., Scolforo, J.R. \& Mello, J.M. 1993. Composição florística e estrutura comunitária de um remanescente de floresta semidecídua montana em Lavras (MG). Rev. Bras. Bot. (no prelo).

Rodrigues, R.R. 1986. Levantamento florístico e fitossociológico das matas da Serra do Japi, Jundiai, SP. Campinas, Universidade Estadual de Campinas, dissertação de mestrado.

Silva, A.F. 1989. Composição florística e estrutura fitossociológica do estrato arbóreo da Reserva Florestal Professor Augusto Ruschi, São José dos Campos, SP. Campinas, Universidade Estadual de Campinas, tese de doutoramento.

Torres, R.B. 1989. Estudos florísticos em mata secundária da Estação Ecológica de Angatuba, Antatuba (São Paulo). Campinas, Universidade Estadual de Campinas, dissertação de mestrado.

Torres, R.B., Martins, F.R. \& Gouvea, L.S.K. 1993. Spatial partition of forest tree species in the state of São Paulo, Southeastern Brazil. J. Biogeog. (no prelo).

Veloso, H.P., Rangel Filho, A.L.R. \& Lima, J.C.A. 1991. Classificação da vegetação brasileira adaptada a um sistema universal. IBGE, Rio de Janeiro.

Vilela, E.A., Oliveira-Filho, A.T., Carvalho, D.A. \& Gavilanes, M.L. 1993. Flora arbustivoarbórea das matas ciliares do Alto Rio Grande (MG). 3 - mata de Camargos, Itutinga. Acta bot. bras. 